\title{
Otomycosis-Clinical and Mycological Spectrum
}

\author{
M. Swetha ${ }^{1}$, H.R. Divya ${ }^{2 *}$ K. Anuradha ${ }^{2}$ and M.K. Veenapani ${ }^{3}$ \\ ${ }^{1}$ Mysore Medical College and Research Institute, Mysore, Karnataka, India \\ ${ }^{2}$ Department of Microbiology, Mysore Medical College and Research Institute, \\ Mysore, Karnataka, India \\ ${ }^{3}$ Department of ENT, Mysore Medical College and Research Institute, \\ Mysore, Karnataka, India \\ *Corresponding author
}

\section{A B S T R A C T}

Otomycosis is an external ear canal infection caused by various fungi. The aim of this study was to identify the fungal agents, predisposing factors and characteristics of patients. Between June 2018 to December 2018, 66 patients with clinical suspicion of otomycosis were enrolled and the samples from their external ear were examined for any mycological infection. Otomycosis was confirmed after mycological diagnosis in 60 of clinically suspected patients. Otomycosis was found to be more common among females (38) and majority in the age-group 30-40 years $(34.84 \%)$. Pruritis was the most commonly presenting symptom seen in $57.5 \%$ of the otomycotic patients. Self cleansing was found to be predominating predisposing factor for Otomycosis $(50 \%)$, followed by Instillation of coconut oil (13.63\%). Aspergillus niger 29(43.93\%) was the predominant species isolated, second most common species isolated was Aspergillus flavus 20(30.3\%), Aspergillus fumigatus 5(7.57\%), Candida species 3(4.54\%), Penicillium species 2(3.03\%) and Mucor $1(1.51 \%)$ were other species isolated. Clinical suspicion of otomycosis is important to prevent unnecessary use of antibiotics. The present study highlights the practice of self cleaning and using home remedies and eardrops to get relief from sensation of blocked ear and itching which are the predominant risk factors for otomycosis.

\section{Introduction}

Otomycosis is a common condition, frequently encountered in otolaryngology outpatient clinics. It is defined as a fungal infection affecting the external ear canal in the majority of cases. Less commonly, it can involve the middle ear, if the drum is perforated, and the mastoid cavity following an open mastoidectomy ${ }^{1}$ Its prevalence has been quoted to range from $9 \%^{2}$ to $27.2 \%{ }^{3,4}$ among patients who present with signs and symptoms of otitis externa and up to $30 \%^{5}$ in patients with discharging ears. It is worldwide in distribution with a higher prevalence in the hot, humid, and dusty areas of the tropics and subtropics $^{3,4,6,7}$. Overview of the literature reveals otomycosis to be a common medical problem in India ${ }^{8,9}$. Fungi can either be the primary pathogen or be superimposed on bacterial infections. Most patients suffering from early otomycosis complain of severe itching which often progress to pain, hearing loss, aural discharge, and often leading to 
tympanic membrane perforations ${ }^{9}$. Although Aspergillus niger and Candida albicans are by far the most common offenders ${ }^{3,6-9}$, a wide spectrum of other fungi can cause otomycosis. Various factors have been proposed as predisposing factors for otomycosis, including a humid climate, presence of cerumen, instrumentation of the ear, self-inflicted trauma (use of q-tips to clean the ear), immunocompromised host, and, more recently, increased use of topical antibiotic/steroid preparations. ${ }^{10}$

The study is taken up to know the various fungi responsible for ear infection and to compare with demographical details.

\section{Materials and Methods}

This study is an institution based prospective study. The ethical clearance was obtained from institutional ethical committee. The subjects included for the study are those who attended ENT OPD with symptoms like itching, pain, feeling of blocked ear, deafness, discharge and otoscopic findings revealing wet or dry matted masses of hyphae/spores or thick, white cheesy material. ${ }^{11,}{ }^{12}$. Patients with otitis externa due to other causes like history of trauma to external auditory canal and prior ear surgery were excluded from the study

The study was conducted during the period of June 2018-December 2018. Total of 66 subjects fulfilling the inclusion criteria was included after taking informed consent. The detailed predesigned proforma was filled and sample was collected with two sterile swabs and sent to the microbiology department.

\section{Sample processing}

Specimens were collected aseptically using two sterile cotton swabs from each diseased ear canal and sent immediately to microbiology laboratory. The samples were processed following standard fungal identification protocol. Of these, one swab was subjected to direct microscopic examination in $10 \% \mathrm{KOH}$ wet mount and the other was inoculated on Sabouraud Dextrose Agar with antibiotic (Gentamicin and Chloramphenicol) in duplicate and incubated at $25^{\circ} \mathrm{C} \& 37^{\circ} \mathrm{C}$ for upto three weeks. The culture tubes were examined for any fungal growth, daily during the first week \& then twice weekly during the next two weeks. Positive cultures were identified by colony morphology and microscopic morphology in Lactophenol Cotton Blue (LCB) mount and Slide culture. All Candida isolates were identified by Germ tube test \& chlamydospore formation on Corn meal agar. $^{13}$

\section{Results and Discussion}

In the present study, out of the total 66 cases of clinically-diagnosed otomycosis, 28 were males and 38 were females (Figure 1). The highest incidence of otomycosis was in the age group of 30-40 years and the lowest was noted in the age group of $<10$ years (Table 1).

In our study majority of patients presented with the symptom of Pruritis 38(57.5\%) followed by Pain 21(31.8\%). Other symptoms were Hearing loss18 (27.2 \%) and ear discharge $8(1.21 \%)$ (Table 2).

Data of the various predisposing factors for otomycosis is given in Table 3. The incidence of otomycosis was high in patients with history of Self cleansing 33 (50\%). The use of unsterile oil in an attempt to clean ear was found in $9(13.63 \%)$ patients, 10(15.15\%) patients were associated with Diabetes and least association was found in subjects with CSOM 6(9.09\%)

Specimen was collected from 66 clinically diagnosed cases of otomycosis out of which 60 specimens yielded growth (Table 4). All 
specimens yielded single organism. flavus 20(30.3\%). Aspergillus fumigatus Aspergillus (81\%) was the commonest species 5(7.57\%), Candida species 3(4.54\%), found. Aspergillus niger 29(43.93\%) was the Penicillium species 2(3.03\%) and Mucor predominant species isolated. Second most species $1(1.51 \%)$ were other species

common species isolated was Aspergillus

Table.1 Age wise distribution of otomycosis patients

\begin{tabular}{|c|c|c|}
\hline Age (in years) & Total & \% \\
\hline$<\mathbf{1 0}$ & 2 & 3.03 \\
\hline $\mathbf{1 1 - 2 0}$ & 5 & 7.57 \\
\hline $\mathbf{2 1 - 3 0}$ & 10 & 15.15 \\
\hline $\mathbf{3 1 - 4 0}$ & 23 & 34.84 \\
\hline $\mathbf{4 1 - 5 0}$ & 11 & 16.16 \\
\hline $\mathbf{5 1 - 6 0}$ & 9 & 13.63 \\
\hline$>\mathbf{6 0}$ & 6 & 9.09 \\
\hline total & 66 & 100 \\
\hline
\end{tabular}

Table.2 Presenting symptoms of cases

\begin{tabular}{|l|l|c|}
\hline Presenting symptom & No of patients & \% \\
\hline Pruritis & 38 & 57.5 \\
\hline Pain & 21 & 31.8 \\
\hline Hearing loss & 18 & 27.2 \\
\hline Discharge & 8 & 1.21 \\
\hline
\end{tabular}

Table.3 Predisposing factors for otomycosis

\begin{tabular}{|l|l|}
\hline Predisposing factor & Frequencies \\
\hline Self cleansing & $33(50 \%)$ \\
\hline Instillation of oil & $9(13.63 \%)$ \\
\hline Instillation of topical ear drops & $8(12.12 \%)$ \\
\hline Diabetes & $10(15.15 \%)$ \\
\hline CSOM & $6(9.09 \%)$ \\
\hline
\end{tabular}

Table.4 Fungal isolates from otomycosis patients

\begin{tabular}{|l|l|}
\hline Fungal isolates & No of patients \\
\hline Aspergillus niger & $29(43.93 \%)$ \\
\hline Aspergillus flavus & $20(30.3 \%)$ \\
\hline Aspergillus fumigatus & $5(7.57 \%)$ \\
\hline Candida species & $3(4.54 \%)$ \\
\hline Penicillium species & $2(3.03 \%)$ \\
\hline Mucor species & $1(1.51 \%)$ \\
\hline No fungal isolate & $6(9.09 \%)$ \\
\hline Total & 66 \\
\hline
\end{tabular}


Fig.1 Sex distribution among cases

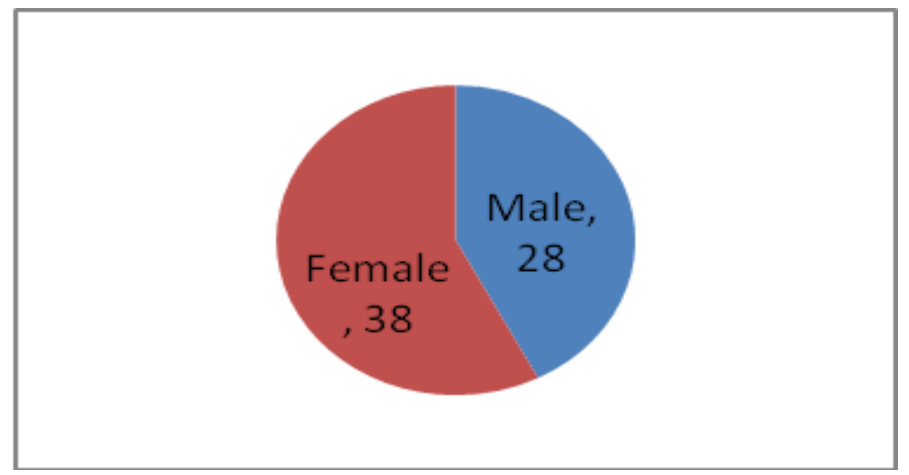

Otomycosis is a significant mycotic infection of external auditory canal frequently encountered by otolaryngologist and can usually be diagnosed by clinical examination. It is highly prevalent in tropical \& subtopical regions of the world. This could be due to hot $\&$ humid weather and presence of dust in the environment, all of which favours the dissemination and growth of fungus.

This study was aimed to assess the relationship between the fungal appearance and clinical symptoms of otomycosis, and to determine most prevalent microbial isolate in otomycosis. A total of 66 (38 males, 28 females) clinically diagnosed cases of otomycosis were included in the study. In our study, females $(57.6 \%)$ were more commonly affected by Otomycosis than males (42.4) this is in agreement with studies by Aneja KR et al and Panchal et al., ${ }^{14,15}$. The percentage of females was $57 \%$ in our study, which may be due to the household work like dusting, cleaning or gardening thus exposing them to the fungal spores.

The age groups between 6-70 years were considered. Incidence was highest in young adults between 30-40 yrs age group. Considering the fact that otomycosis can occur at any age, the common age groups affected were $21-30$ years $(15.15 \%)$ and $31-$ 40 years $(34.84 \%), 44-50$ years $(13.63 \%)$ i.e. adults which constituted the working population. This observation is in accordance with Desai KJ et al., Nandyal CB et al and most other studies from India. ${ }^{16,}$. The people in age group from 11-40 years usually spend more time in the outdoors and are more exposed to the fungal spores due to occupational exposure, travelling etc making them more vulnerable to otomycosis.

In the present study, the predominant predisposing factor for Otomycosis was found to be self cleansing $(50 \%)$. Metallic /wooden/paper roll etc commonly used for cleaning ear canal, often leads to trauma of the canal skin into which the fungal spores may seed in. Moreover it damages the normal lining epithelium, which is the natural defense against such infections. This is in accordance with the study conducted by Pontes et al., ${ }^{18}$

In our study second most common predisposing factor is oil instillation (13.63\%) into the external ear and association of Diabetes (15.15\%). Other predisposing factors were chronic suppurative otitis media and prior use of topical antibiotic eardrops. Oils have fatty acids that provides a suitable medium for the growth of fungus, which explains the higher incidence of otomycosis in people who instill oils regularly. Recurrent use of antibiotic drops, steroids, antiseptics or wax solvent ear drops applications alters the local environment of the external ear canal and allows super infection by fungus. This is 
in accordance with the study conducted by Pontes et al., ${ }^{18}$.

In our study itching in the ear was the commonest symptom in $57.5 \%$ of the patients followed by pain (31.8\%) in ear and hearing loss $(27.2 \%)$, similar findings was found in other studies ${ }^{19}$. The fungus growth mixed with the epithelial debris and cerumen forms characteristic mycotic plug which gives rise to the symptom of blocked ear and hearing loss.

In our study, Aspergillus niger (43.9\%) was the most commonly isolated organism. This finding is similar to what was observed by other studies 20,21 . A. flavus $(30.3 \%)$ was found to be the second most common causative agent followed by A. fumigates (7.5\%). Other fungi that have been associated with otomycosis in our study were Candida, Mucor and Penicillium sp. However, the results obtained by Kaur et al., ${ }^{22}$. show that in the tropical regions, the commonest aetiologic agents accountable for otomycosis are A. fumigates $(41.1 \%)$ and A. niger $(36.9 \%)$.

Aspergillus species and Candida species are the most commonly identified fungal pathogens in Otomycosis ${ }^{23}$. Aspergilli have an optimum $\mathrm{pH}$ range of 5.7 and a maximum growth rate at a temperature of $37^{\circ} \mathrm{C}$ and this is conducive for all species of Aspergillus isolated in the present study. This is supported by the predilection of fungi to grow in the inner one-third of external auditory canal ${ }^{10,24}$.

In conclusion, otomycosis is fungal infection of the EAC that is frequently encountered in patients attending otolaryngology clinics. As clinical features are non specific, this study demonstrates the laboratory diagnosis of otomycosis and its confirmation by fungal culture which helps in tackling the disease. Clinical suspicion of otomycosis can also prevent unnecessary use of antibiotics.
In the present study, the predominant predisposing factor for Otomycosis was found to be self cleansing (50\%). Metallic /wooden/paper roll etc commonly used for cleaning ear canal, followed by common predisposing factor is oil instillation (13.63\%) often leads to trauma of the canal skin damaging the normal lining epithelium into which the fungal spores may seed in. Constant use of ear buds and unnecessary usage of ear drops may be avoided as they are the predominant risk factors for otomycosis. Educating the patients about risk factors, impact of otomycosis and its complications will help to reduce the disease burden.

\section{References}

1. Khaled Ali, Mahmood A. Hamed, Hameda Hassan, Identification of Fungal Pathogens in Otomycosis and Their Drug Sensitivity: Our Experience Int Arch Otorhinolaryngol 2018; 22: 400-403.

2. Mugliston, T., and G. O'Donoghue, "Otomycosis-a continuing problem," Journal of Laryngology and Otology, vol. 99, no. 4, pp.327-333, 1985.

3. Pontes, Z. B. V. D. S., A. D. F. Silva, E. D. O. Lima et al., "Otomycosis: a retrospective study," Brazilian Journal of Otorhinolaryngology, vol. 75 , no. 3, pp. 367-370, 2009.

4. Fasunla, J., T. Ibekwe, and P. Onakoya, "Otomycosis in western Nigeria," Mycoses, vol. 51, no. 1, pp. 67-70, 2008.

5. Kurnatowski, P., and A. Filipiak, "Otomycosis: prevalence, clinical symptoms, therapeutic procedure," Mycoses, vol. 44, no. 11-12, pp. 472-479, 2001.

6. Pradhan, B., N. RatnaTuladhar, and R. Man Amatya, "Prevalence of otomycosis in outpatient department of otolaryngology in Tribhuvan University Teaching Hospital, Kathmandu, Nepal," Annals of Otology, Rhinology and Laryngology, vol. 112,no. 4, pp. 384-387, 2003.

7. R. Munguia and S. J. Daniel, "Ototopical antifungals and otomycosis: a review," International Journal of Pediatric 
Otorhinolaryngology, vol. 72, no. 4, pp. 453-459, 2008.

8. R. Kaur, N. Mittal, M. Kakkar, A. K. Aggarwal, and M. D. Mathur, "Otomycosis: a clinicomycologic study," Ear, Nose and Throat Journal, vol. 79, no. 8, pp. 606-609, 2000.

9.B. Viswanatha, D. Sumatha, and M. S. Vijayashree, "Otomycosisin immune competent and immunocompromised patients: comparative study and literature review," Ear, Nose \& Throat Journal, vol. 91, pp. 114-121, 2012.

10.Sampath Chandra Prasad,1 Subbannayya Kotigadde,2 Manisha Shekhar,3Primary Otomycosis in the Indian Subcontinent: Predisposing Factors, Microbiology, and Classification Hindawi Publishing Corporation; International Journal of Microbiology, Volume 2014, Article ID 636493, 9 pages

11. Collee JG, Miles RS, Watt B. Tests for identification of bacteria. In: Mackie and [11] McCartney's Practical Medical Microbiology, Collee JG, Fraser AG, Marmion BP, Simmons A, Eds Churchill Livingstone, New York, NY, USA; 1996; pp. 131-45.

12. Chander J. Textbook of Medical Mycology. 3rd Edition, New Delhi, Mehta [12] Publishers; 2002.

13. F. Fischer and MB Cook, "Some opportunistic fungi and yeasts and yeast-like fungi," in Fundamentals of Diagnostic Mycology, F. Fischer and M. B. Cook, Eds., pp. 35-225, W.B. Saunders, Philadelphia, Pa, USA, 1998.

14. Aneja KR, Sharma C, Joshi R. (2010). Fungal infection of the ear: a common problem in the north eastern part of Haryana. Int $\mathbf{J}$ Pediatr Otorhinolaryngol., 74(6), 604-7.
15. Panchal P, Pethani J, Patel D, Rathod S, Shah P. (2013). Analysis of various fungal agents in clinically suspected cases of otomycosis. Indian $\mathbf{J}$ of Basic \& Applied Medical Research, 2(8), 865-869.

16. Desai KJ, Malek SS, Italia IK, Jha S, Pandya V, Shah H. (2012). Fungal Spectrum In Otomycosis At Tertiary Care Hospital. NJIRM, 3(5), 58-61.

17. Nandyal CB, Choudhari AS, Sajjan NB. (2015). A Cross sectional study for Clinicomycological Pro $\square$ le of Otomycosis in North Karnataka. Int J Med Health Sci., 4(1), 64-69.

18. Pontes ZB, Silva AD, Lima Ede O, Guerra Mde H, Oliveira NM, CarvalhoMde F, Guerra FS Otomycosis; A retrospective study. Braz J of Otolarygology 2009; 75: 367-70.

19. T. Dinesh Singh, C. P. Sudheer; Otomycosis: a clinical and mycological studyInt $J$ Otorhinolaryngol Head Neck Surg. 2018 Jul; 4(4): 1013-1016

20. Ghiacei S. Survey of Otomycosis in northwestern area of Iran. Med J Mashhad Uni Med Sci 2001; 43: 85-7.

21. Sephidgar A, Kyakajouri K, Meyrzaei M, Sharifi F. Fungal infection of external ear in otomycosis. J Babol Med Sci 2001; 13:25-9.

22. Kaur R, Mittal N, Kakkar M, Aggarwal AK, Matthur. MD. Otomycosis: a clinicomycologic study. Ear Nose Throat J. 2000; 79:606-9.

23. Martin TJ, Kerschner JE, FlanaryVA.. Fungal causes of otitis externa and tympanostomy tube otorrhea. International Journal of pediatric Otorhino laryngology.2005; 96: 1503- 8

24. 25 Pahwa VK, Chamiyal PC, Suri PN.Mycological study in otomycosis. Indian J Med Res 1983; 77: 334-8.[6874014]

\section{How to cite this article:}

Swetha, M., H.R. Divya, K. Anuradha and Veenapani' M.K. 2019. Otomycosis-Clinical and Mycological Spectrum. Int.J.Curr.Microbiol.App.Sci. 8(06): 2845-2850. doi: https://doi.org/10.20546/ijcmas.2019.806.343 